\title{
BMJ Open Healthcare resource availability and cardiovascular health in the USA
}

\author{
Courtney S Pilkerton, ${ }^{1}$ Sarah S Singh, ${ }^{2}$ Thomas K Bias, ${ }^{3}$ Stephanie J Frisbee ${ }^{4}$
}

To cite: Pilkerton CS, Singh SS, Bias TK, et al. Healthcare resource availability and cardiovascular health in the USA. BMJ Open 2017;7:e016758. doi:10.1136/ bmjopen-2017-016758

- Prepublication history and additional material for this paper are available online. To view these files, please visit the journal online (http://dx.doi. org/10.1136/bmjopen-2017016758).

This work was presented, in part, at the American Heart Association Epidemiology and Prevention/Nutrition, Physical Activity and Metabolism 2014 Scientific Sessions in San Francisco, California, USA.

Received 11 March 2017 Revised 5 October 2017 Accepted 6 October 2017

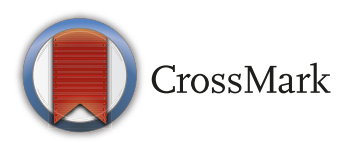

${ }^{1}$ Department of Family Medicine, School of Medicine, West Virginia University, Morgantown, West Virgina, USA

${ }^{2}$ Department of Epidemiology \& Biostatistics, Schulich School of Medicine \& Dentistry, University of Western Ontario, London,

Ontario, Canada

${ }^{3}$ Department of Health Policy, Management \& Leadership,

School of Public Health, West Virginia University, Morgantown, West Virginia, USA

${ }^{4}$ Departments of Pathology \& Laboratory Medicine, and Epidemiology \& Biostatistics, Schulich School of Medicine \& Dentistry, University of Western Ontario, London, Ontario, Canada

Correspondence to Dr Stephanie J Frisbee; sfrisbee@uwo.ca

\section{ABSTRACT}

Objectives Cardiovascular disease (CVD) remains the leading cause of death in the USA. Reducing the population-level burden of CVD disease will require a better understanding and support of cardiovascular health (CVH) in individuals and entire communities. The objectives for this study were to examine associations between community-level healthcare resources ( $\mathrm{HCrRes)}$ and $\mathrm{CVH}$ in individuals and entire communities.

Setting This study consisted of a retrospective, crosssectional study design, using multivariable epidemiological analyses.

Participants All participants in the 2011 Behavioral Risk Factor Surveillance System (BRFSS) survey were examined for eligibility. CVH, defined using the American Heart Association CVH Index (CVHI), was determined using self-reported responses to 2011 BRFSS questions. Data for determining HCrRes were obtained from the Area Health Resource File. Regression analysis was performed to examine associations between healthcare resources and $\mathrm{CVHI}$ in communities (linear regression) and individuals (Poisson regression).

Results Mean CVHI was $3.3 \pm 0.005$ and was poorer in the Southeast and Appalachian regions of the USA. Supply of primary care physicians and physician assistants were positively associated with individual and communitylevel CVHI, while CVD specialist supply was negatively associated with CVHI. Individuals benefiting most from increased supply of primary care providers were: middle aged; female; had non-Hispanic other race/ethnicity; those with household income $<\$ 25000 / y e a r ;$ and those in nonurban communities with insurance coverage.

Conclusions Our results support the importance of primary care provider supply for both individual and community CVHI, though not all sociodemographic groups benefited equally from additional primary care providers. Further research should investigate policies and factors that can effectively increase primary care provider supply and influence where they practice.

\section{INTRODUCTION}

Although mortality attributable to cardiovascular disease (CVD) continues to decline, CVD remains the cause of one-third of all deaths in the USA ${ }^{1}$ and it is projected that, by 2030 , more than $40 \%$ of Americans will be living with CVD and, further, the direct medical costs of CVD are predicted to triple and indirect costs to increase by $61 \%$ (both from 2010 levels). ${ }^{2}$ To ameliorate the burden
Strengths and limitations of this study

- Used Behavioral Risk Factor Surveillance System and other census-derived and nationally representative datasets.

- This study examined the association between healthcare resources (provider supply and physical facilities) and cardiovascular health, as defined by the American Heart Association's cardiovascular health index, in both individuals and populations.

- Thus, our results reported here provide strong support that increasing the supply of primary care providers is likely to improve cardiovascular health in individuals and entire communities.

- Two key caveats are noted: not all sociodemographic groups will be likely to benefit equally from additional primary care providers, and there are significant challenges to increasing the number of primary care providers in communities.

- To our knowledge, this is the first study to assess the relationship between healthcare resource availability and cardiovascular health. A key strength of this study is the use of Behavioral Risk Factor Surveillance System (BRFSS)-a large, nationally representative sample. The key, and wellrecognised, limitation of this study is the self-report methodology for all BRFSS questions, which may have overestimated the cardiovascular health index.

of CVD, improving cardiovascular health $(\mathrm{CVH})$ has been prioritised as a public health goal. Both the American Heart Association (AHA), through their 2020 Strategic Impact Goals, ${ }^{3}$ and the CDC, through Healthy People 2020 (HP2020) objectives, ${ }^{4}$ aim to improve the CVH of Americans.

To advance these priorities, the AHA developed a comprehensive index to measure CVH in individuals and populations. ${ }^{3}$ The scientific rationale as well as the development process and criteria for this score have been thoroughly described elsewhere. ${ }^{3}$ The cardiovascular health index (CVHI) is a composite of seven well-recognised and evidence-based CVD risk factors that includes both biological health factors (total cholesterol, blood pressure, body mass index and blood glucose) and health behaviours (smoking, physical activity and diet). The 
CVHI is intentionally not a predictive score, but rather an aggregate measure of $\mathrm{CVH}$. Thus, each of the seven elements is weighted evenly and scored according to age and gender-specific criteria constituting 'ideal' status for that element (two points), 'intermediate' status for that element (one point) or 'poor' status for that element $(0$ points), resulting in a total score that can range from 0 points ('poor' on all seven elements) to 14 points ('ideal' on all seven elements). Because the scoring criteria have been established to be age and gender specific, including for children, importantly everyone in a population (or sample) receives a non-binary, numeric score, which affords important analytic advantages to the CVHI. Also part of the design of the CVHI, the index can be used directly by individuals (specifically, the 'My Life Check - Life's Simple Seven' campaign by the American Heart Association $^{5}$ ), by healthcare providers, or can be applied to populations and epidemiological cohorts, such as nationally representative and longitudinal studies, so as to advance health promotion, research, population-level monitoring, and, ultimately, policies and approaches to improve CVH in individuals and entire communities.

Because of the importance and flexibility of this tool, the CVHI has been widely used and applied in now hundreds of studies. Generally, the prevalence of 'ideal' CVHI has been reported to be quite low-estimates from nationally representative US studies have ranged from $1 \%$ to $5 \% .{ }^{67}$ Significant racial and ethnic disparities in ideal CVH have also been reported. ${ }^{8}$ With growing concern for the ability to meet stated public health goals, ${ }^{9}$ the need for population-based approaches is underscored. Such approaches will require an understanding of $\mathrm{CVH}$, factors that affect $\mathrm{CVH}$ and the tools, such as public policies, ${ }^{10}$ that can improve $\mathrm{CVH}$ in individuals and entire populations.

Healthcare resources (HCrRes) are understood as key to maintaining and improving health, as reflected in HP2020 objectives to increase access to insurance, providers, preventive services and medical homes. ${ }^{4} \mathrm{~A}$ medical home has been associated with better health status, ${ }^{11}$ more equitable care ${ }^{12}$ and increased use of preventive services. ${ }^{13} 14$ Two essential dimensions of a medical home are availability (supply) of providers and physical facilities.

Increased physician supply has been associated with better outcomes across myriad medical conditions and procedures, ${ }^{15}$ and was found to result in improved health outcomes. ${ }^{16}$ Additionally, increased primary care physician supply (PCP-S) has been associated with increases in positive health outcomes, ${ }^{11}{ }^{12}{ }^{17}$ including self-reported general health, ${ }^{18}$ higher state health rankings ${ }^{19}$ and decreased mortality. ${ }^{20}$ One study determined that a 1 per 10000 population increase in PCP-S decreased all-cause mortality $5.3 \%$ per year. ${ }^{21}$ As there is a documented shortage of PCP-S, particularly in rural areas, ${ }^{22}$ it has been proposed that increasing the availably of physician assistants (PA) and nurse practitioners (NP) may compensate. ${ }^{23}$ Several studies have suggested that PAs and NPs provide a similar quality of services and may contribute to cost-containment, ${ }^{24}$ particularly in rural areas. ${ }^{25} 26$

Community health centres, such as Rural Health Centres (RHC) and Federally Qualified Health Centres (FQHC) funded by the Health Research and Services Administration (HRSA), aim to provide a medical home to underserved communities and vulnerable populations. ${ }^{27}{ }^{28}$ Uninsured and Medicaid patients visiting these centres are more likely to have a regular source of care, have seen a provider in the past year and to receive preventive screenings when compared with patients at other sites of care. ${ }^{29}$ Further, studies have reported that these centres provide more equitable care and an increased number of services for vulnerable populations when compared with other primary care sites. ${ }^{30} 31$

The purpose of this study was to examine the association between HCrRes (provider supply and physical facilities) and CVH, as defined by the CVHI, in both individuals and populations. Thus, we conducted a cross-sectional, multivariable analysis. Necessary adjustment for the insurance status of individuals and communities was considered, but was not the focus of this study.

\section{METHODS}

\section{Participants}

The Behavioral Risk Factor Surveillance System (BRFSS), administered by the CDC, is an annual telephone survey administered in each US state and the District of Columbia, collecting information on health behaviours, chronic conditions and use of preventive services. ${ }^{32}$ Random digit dialling and a complex sampling frame are used to interview adults $\geq 18$ years of age who are part of the civilian, non-institutionalised population. Commencing in 2011, both landline and cell phone numbers were included in the BRFSS sampling frame. Detailed descriptions of the BRFSS study design and methods are described elsewhere. ${ }^{32}$ Due to question availability for all AHA CVHI components, 2011 BRFSS data were used in this study.

All 2011 BRFSS participants were examined for eligibility $(n=507402)$. Participants were ineligible if they were missing data necessary to calculate any of the CVHI components $(n=157908)$, or a county Federal Information Processing Standards (FIPS) code $(\mathrm{n}=37163)$. Females were also ineligible if they reported being pregnant (or unknown) at the time of survey $(n=3693)$.

Communities were defined as a health service area (HSA). HSAs, originally defined in the 1990s based on the hospital usage patterns of Medicare recipients, ${ }^{33}$ are a single county or cluster of contiguous counties which are reasonably independent regarding hospital care. Unique county FIPS codes were aggregated to a unique HSA using the National Cancer Institute's Surveillance, Epidemiology, and End Results Program. ${ }^{34}$ HSAs with fewer than 15 eligible participants were excluded $(n=120)$.

The final, eligible population included in this study was 308895 individuals (60.9\% of all 2011 respondents) from 833 HSAs $(87.5 \%$ of all HSAs). 


\section{Outcome measure (dependent variable): CVHI}

Each of the seven CVHI components (blood pressure, total cholesterol, blood sugar, body mass index, smoking, physical activity, nutrition) was assigned points, with points summed for an overall score. ${ }^{3}$ As we, and others, have previously reported, the original CVHI scoring method must be adapted slightly, as BRFSS questions only permit the determination of two levels for each factor. ${ }^{635}$ As outlined in online Supplemental table S1, CVHI was calculated as a count of components meeting 'ideal' criteria and could range from 0 to 7 , with higher scores indicating better CVH.

\section{Exposure variables (independent variables): HCrRes}

Data for determining HCrRes were obtained from the Area Health Resource File (AHRF), ${ }^{36}$ an annual compilation of healthcare and socioeconomic data from $>50$ sources that is amassed and maintained by HRSA. We defined HCrRes as the number of primary care physicians, PAs, NPs and CVD specialists; and the number of hospital beds, FQHCs or RHCs, and hospitals with a primary care department. The technical definition and primary source for each variable is available in the documentation accompanying the AHRF. ${ }^{36}$ Estimates of resource availability were determined by summing the absolute number of each resource for all counties in an HSA and then dividing by the total HSA population.

\section{Covariates}

For individuals, demographic characteristics obtained from BRFSS included age; sex; race/ethnicity; education level; income; and insurance status. Sociodemographic characteristics of each county were obtained from the $\mathrm{AHRF}^{36}$ and aggregated to each HSA as discussed above. HSA-level covariates included were (for categorical variables, expressed as a percentage of the total HSA population) male; non-Hispanic black; Hispanic; median household income; 4-year college graduates; health insurance status; urban status; aged 65+; poverty. Data availability in the AHRF varies slightly, so data for covariates ranged from 2010 or 2011. However, intracommunity year-to-year variation in these characteristics is small; the impact on the results of subsequent analyses is expected to be negligible.

\section{Statistical analysis}

This study consisted of a retrospective, cross-sectional study design. For individuals, Poisson regression analyses were performed to determine the association between HCrRes and individual CVHI, with adjustment for HSA and individual-level covariates. Poisson regression coefficients were interpreted as mean ratios. The assumptions for Poisson regression were assessed both within and outside of survey procedures and, for all models, test statistics indicated that overdispersion was not present or unlikely (test results not shown).

Community-level demographic characteristics were estimated using means and percentages of each covariate, and age-standardised mean CVHI was determined using 2000 US projected population (distribution 8). ${ }^{37}$ Linear regression analyses were performed to assess the association between HCrRes and CVHI in communities (HSAs). Standardised coefficients were examined to assess the comparative impact of HCrRes and covariates on HSA-level CVHI. Assumptions for linear regression analysis were assessed and, for all models, assumptions were satisfied (test results not shown).

Interactions between PCP-S and all individual and HSA-level covariates were assessed. For significant interactions, we determined the predicted CVHI at given numbers of primary care physicians for each covariate strata, with all other covariates in the model set to the mean level for that covariate.

For community-level analyses, results for descriptive statistics are presented as mean $\pm \mathrm{SD}$, or as frequencies with the corresponding proportion. All analyses of individual-level data were conducted using survey procedures to account for BRFSS survey weights and sampling design and thus results from all individual-level analyses are reported as weighted results. Due to the sampling methodology employed by BRFSS, estimates for SD for individual-level analyses may not be accurate and thus all weighted mean values are reported \pm SE.

Mapping of CVHI in HSAs was performed using ArcGIS 10.1 (ESRI 2012. ArcGIS Desktop: Release 10.1. Redlands, California: Environmental Systems Research Institute). All statistical analyses were performed with Stata V.13 (Stata2013. Stata Statistical Software: Release 13. College Station, Texas: Stata).

\section{RESULTS}

Individual and community (HSAs) characteristics are presented in table 1. Eligible individuals were more likely to be female $(52.5 \%)$, non-Hispanic white $(72.4 \%)$, have at least some college education (59.4\%) and have some health insurance coverage $(88.3 \%)$. Compared with eligible individuals, non-eligible individuals were younger, male and less educated (not shown). Included communities were mostly urban $(74.8 \%)$, and averaged $21.4 \% \pm 7.9$ college graduates, $14.4 \% \pm 4.2$ with no health insurance and $15.1 \% \pm 3.5 \geq 65$ years old. Compared with included HSAs, excluded HSAs were more likely to have a smaller non-Hispanic black and Hispanic population, a smaller proportion of college graduates, a slightly larger proportion living in poverty and were substantially more rural (not shown).

Exposure (HCrRes) and outcome variables are also summarised in table 1 . On average, communities had $63.4 \pm 20.8$ per 100000 population primary care physicians and $24.6 \pm 16.0$ per $100000 \mathrm{PAs}$, whereas there were $4.1 \pm 4.0$ per 100000 CVD specialists. Additionally, communities had, on average, $334.0 \pm 193.5$ per 100000 hospital beds. The average CVHI score for individuals was $3.30 \pm 0.005$ units. Mean community-level CVHI was $3.34 \pm 0.3$ units. The geographical distribution of age-standardised 
Table 1 Summary characteristics of the study populations: (A) demographic and socioeconomic characteristics of included individuals; (B) demographic and socioeconomic characteristics of included communities; (C) community (HSA) healthcare resource variables per 100000 population; (D) cardiovascular health for included individuals and communities

A. Individual-level covariates: individual demographic and socioeconomic characteristics

$\begin{array}{lc}\text { Age (years) } & 51.4 \pm 0.06 \\ \text { Sex } & \\ \text { Female } & 189044(52.5 \%) \\ \text { Male } & 119753(47.5 \%) \\ \text { Race/ethnicity } & \\ \text { Non-Hispanic white } & 249022(72.4 \%) \\ \text { Non-Hispanic black } & 25905(11.3 \%) \\ \text { Hispanic } & 15736(11.0 \%) \\ \text { Other } & 10111(5.3 \%) \\ \text { Education } & \\ \text { Less than high school } & 23284(12.2 \%) \\ \text { High school } & 86991(28.4 \%) \\ \text { Some college } & 198083(59.4 \%) \\ \text { Income } & \\ \text { Under } \$ 25000 & 75356(26.6 \%) \\ \text { \$25 000-\$49999 } & 72534(25.2 \%) \\ \text { \$50 000-\$74999 } & 44871(16.3 \%) \\ \text { \$75000 or more } & 79975(31.8 \%) \\ \text { Insurance status } & 283666(88.3 \%) \\ \text { Some coverage } & 24636(11.7 \%) \\ \text { None } & \end{array}$

B. Community-level covariates: community (HSA) demographic and socioeconomic characteristics Age

$\% 65$ years and older

$15.1 \pm 3.5$

Sex

$\%$ Male

$49.6 \pm 1.3$

Race/ethnicity

$\%$ Non-Hispanic black

$9.7 \pm 13.0$

$\%$ Hispanic

$9.4 \pm 12.7$

Education

$\%$ College graduates

$21.4 \pm 7.9$

Income

Median household income (\$)

$\%$ Poverty

$44082 \pm 9958$

$16.7 \pm 5.1$

Health insurance

$\%$ No health insurance $\quad 14.4 \pm 4.2$

Population density

Urban

$623(74.8 \%)$

Not urban

$210(25.2 \%)$
Table 1 Continued

C. Exposure variables: community (HSA) healthcare resources (per 100000 population)

Primary care physicians

$63.4 \pm 20.8$

Physician assistants

$24.6 \pm 16.0$

Nurse practitioners

$40.1 \pm 18.7$

Cardiovascular disease specialists

$4.1 \pm 4.0$

Hospital beds

$334.0 \pm 193.5$

Number of FQHCs or RHCs

$6.6 \pm 7.0$

Hospitals with a primary care

$1.0 \pm 1.4$ department

\section{Outcome measure: CVHI}

Individual $\mathrm{CVHI}$

$3.30 \pm 0.005$

Community CVHI

$3.34 \pm 0.3$

For individual-level demographic characteristics (section A): values are presented as weighted mean \pm SE for continuous variables (age), and unweighted (raw) frequency (n) and weighted proportion (\%) for categorical variables (sex, race/ethnicity, education, income, insurance status) for the entire population of included individuals.

For community-level (HSA) demographic characteristics (section $B$ ): values are presented as mean $\pm S D$, where the statistic has been averaged across all included HSAs. The exception is population density, which is reported as a direct count of all HSAs in each category.

For community-level healthcare resources (section C): values are presented as means $\pm \mathrm{SD}$, where the statistic has been averaged across all included HSAs. Results are reported as per 100000 population.

For CVHI (section D): values for individual-level analysis are presented as the survey weighted mean \pm SE. Values for community-level analysis are presented as age-standardised mean $\pm S D$, where the statistic has been averaged across all included HSAs. For CVHI, higher scores indicate better cardiovascular health. The maximum possible score, for both individuals and aggregated at the HSA, is 7 .

CVHI, cardiovascular health index; FQHC, Federally Qualified Health Centre; HSA: health service area; RHC, Rural Health Centre.

HSA-level CVHI is shown in figure 1. HSAs with insufficient data were more frequently located in the Midwest and upper plains.

Results from Poisson regression analyses assessing the association between HCrRes and individual-level CVHI are shown in table 2. In univariate analysis, all HCrRes variables were associated with CVHI, except NPs. After adjustment for all covariates (individual level and community level), PCP-S, PAs, CVD specialists and hospital beds remained statistically significantly associated with individual-level CVHI: PCP-S and PAs were positively associated with CVHI, while CVD specialists and hospital beds were negatively associated with CVHI.

Results from linear regression analyses assessing the association between community HCrRes and community-level CVHI are shown in table 3. In univariate analysis, all HCrRes variables were associated with CVHI except NPs and hospitals with primary care 


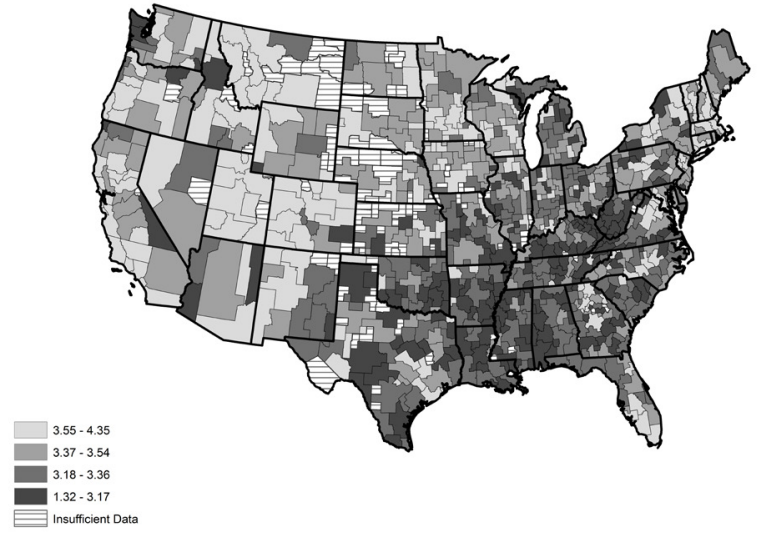

Figure 1 Mean age-standardised cardiovascular health index for US health service area, 2011 (Behavioral Risk Factor Surveillance Survey data).

departments. After adjustment for HSA-level sociodemographic covariates, PCP-S, PAs and CVD specialists remained statistically significantly associated with community CVHI: PCP-S and PAs were positively associated with CVHI, while CVD specialists were negatively associated with community CVHI. Using standardised coefficients, college education was more important than any HCrRes or other HSA-level factor, and the number of primary care physicians was the most important HCrRes associated with community CVHI.

In assessing for differential associations between the number of primary care physicians and individual-level predicted CVHI, we observed statistically significant interactions between PCP-S and the following individual-level covariates: $\operatorname{sex}(p=0.01)$; race/ethnicity $(p=0.04)$; household income $(p=0.001)$; age $(p<0.001)$; community-level health insurance coverage $(p=0.002)$; and population density $(p=0.04)$. Figure 2 presents the predicted individual CVHI for these interactions. As PCP-S increased, CVHI increased in those aged 31-65 years (figure 2A), but decreased in those 18-30 years, and did not change in those $>65$ years. At all income levels, increased PCP-S was associated with higher CVHI, though those with household incomes $<\$ 25000$ benefited most (figure 2B). Increased PCP-S was associated with higher CVHI for all race/ethnic groups except non-Hispanic blacks (figure 2C); 'other' race/ ethnicities benefited the most. Both men and women had higher CVHI with increased PCP-S, but women appeared to benefit more (figure 2D). At all levels of community-level health insurance coverage, higher PCP-S was associated with increased CVHI (figure 2E), though individuals in communities with lower levels of health insurance benefited the most. Individuals in both urban and non-urban communities experienced higher CVHI with increased PCP-S (figure 2F), with individuals in non-urban communities benefiting more than urban-dwelling individuals.

Finally, in community-level models, we observed a near statistically significant interaction $(p=0.07)$ between PCP-S supply and population density, but no statistically significant interactions with any other covariates. As shown in online supplementary figure S1, similar to the results seen for individual-level models, both urban and non-urban communities had increased CVHI with increased PCP-S, and non-urban communities benefited slightly more than urban communities.

\section{DISCUSSION}

\section{Major findings and interpretation}

To our knowledge, this is the first study to assess the relationship between HCrRes availability and CVH. Our key finding is that the availability of primary care physicians and PAs is associated with improved CVH in both individuals and entire communities. This observation is consistent with previous studies reporting that PCP-S was associated with better health outcomes and that PAs may provide care similar to physicians. ${ }^{38}$ However, we did not observe an association between NPs and CVHI, though other studies have suggested that NPs may also provide care similar to primary care physicians. ${ }^{24}{ }^{25}$ In this study, we were not able to account for the practice focus of NPs or PAs. Futures studies that differentiate between primary care-focused care and specialty-focused care may result in additional insights into the role of NPs and PAs inCVH.

Our observation that CVD specialist supply was negatively associated with CVHI is consistent with Starfield $e t$ $a l,{ }^{12}$ who reported that specialists were associated with increased mortality. These observations are not unexpected in a cross-sectional study: hospitals, and the specialists who staff them, tend to be geographically concentrated in areas of higher population and sicker individuals may move to areas with the HCrRes they need. The complex relationships between PCP-S, specialists, hospitals, the quality of care and health outcomes have been discussed by others,${ }^{16}$ and require further study.

HCrRes, particularly PCP-S, community covariates and individual factors were all significantly associated with individual-level CVHI, though individual factors had the greatest influence. This is not unexpected, as individual factors are much more proximal to individual health than are community factors. Further, these results are consistent with a deep literature discussing the positive association between education and health. ${ }^{39}$ Interestingly, our results suggest that an individual's insurance status was not associated with CVHI, which is consistent with Sox et $a l,{ }^{40}$ who reported that a regular physician was more important than insurance status, as well as a more recent report from National Health and Nutrition Examination Surveys data wherein the observed association between insurance status and 'ideal' CVHI did not withstand adjustment for socioeconomic status. ${ }^{41}$ 
Table 2 Results from Poisson regression analysis assessing the association (mean ratios) between community (HSA) healthcare resources and individual-level CVHI, unadjusted and adjusted for individual and community (HSA) socioeconomic and demographic covariates

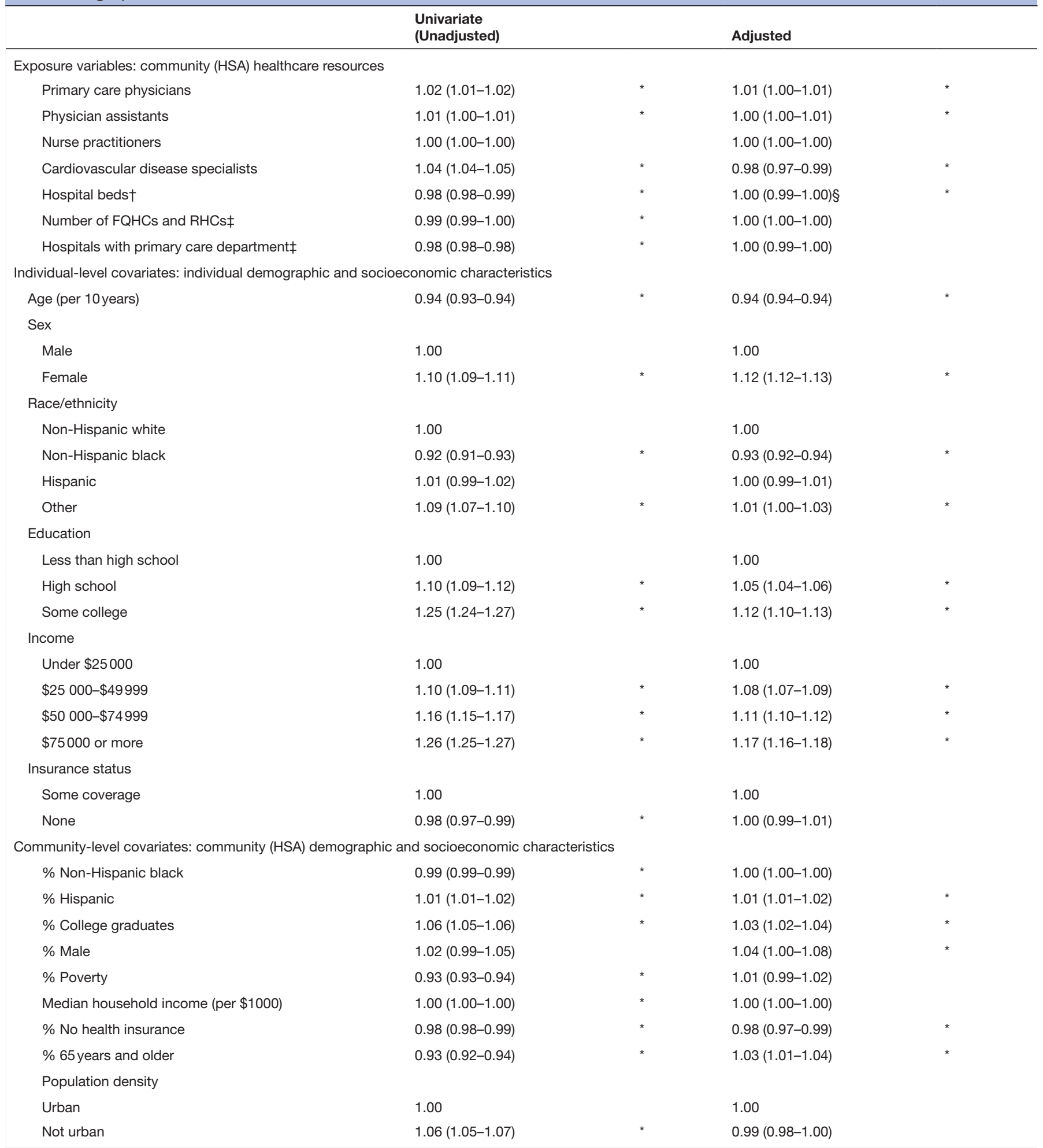

For all variables, coefficients are presented as a 10-unit change in the covariate unless otherwise specified.

*Indicates statistical significance at the alpha 0.05 level.

†A 100-unit change ('hospital beds'),

$\ddagger A$ one-unit change ('number of FQHCs and RHCs' and 'hospitals with a primary care department').

§Result presented after rounding (before rounding coefficient was 0.99995 (0.99993-0.99998)).

CVHI, cardiovascular health index; FQHC, Federally Qualified Health Centre; HSA: health service area; RHC, Rural Health Centre. 
Table 3 Results from linear regression analysis assessing the association between community (HSA) healthcare resources and community CVHI, unadjusted and adjusted for community (HSA) socioeconomic and demographic factors

\begin{tabular}{|c|c|c|c|c|c|c|c|}
\hline Covariate & \multicolumn{3}{|c|}{ Univariate } & \multicolumn{2}{|l|}{ Adjusted } & \multicolumn{2}{|l|}{$\begin{array}{l}\text { Standardised } \\
\text { regression } \\
\text { coefficient } \\
\text { ('Beta') }\end{array}$} \\
\hline \multicolumn{8}{|c|}{ Community (HSA) healthcare resources (per 100000 population) } \\
\hline Primary care physicians & 0.06 & 0.05 to 0.07 & * & 0.03 & 0.01 to 0.04 & 0.06 & * \\
\hline Physician assistants & 0.05 & 0.03 to 0.06 & * & 0.01 & 0.003 to 0.03 & 0.02 & * \\
\hline Nurse practitioners & 0.005 & -0.006 to 0.02 & & -0.01 & -0.02 to 0.002 & -0.02 & \\
\hline Cardiovascular disease specialists & 0.12 & 0.07 to 0.18 & * & -0.08 & -0.15 to -0.02 & -0.03 & * \\
\hline Hospital beds $\dagger$ & -0.01 & -0.02 to -0.001 & * & -0.003 & -0.01 to 0.007 & -0.006 & \\
\hline Number of FQHCs and RHCsł & -0.009 & -0.01 to -0.0006 & * & -0.002 & -0.005 to 0.0007 & -0.01 & \\
\hline Hospitals with primary care departmentł & -0.003 & -0.02 to 0.01 & & -0.006 & -0.02 to 0.008 & -0.008 & \\
\hline \multicolumn{8}{|l|}{ Community (HSA) demographic characteristics } \\
\hline$\%$ Non-Hispanic black & -0.05 & -0.07 to -0.04 & * & -0.006 & -0.02 to 0.01 & -0.008 & \\
\hline \% Hispanic & 0.03 & 0.01 to 0.04 & * & 0.03 & 0.009 to 0.05 & 0.04 & * \\
\hline$\%$ College graduates & 0.19 & 0.17 to 0.22 & * & 0.12 & 0.09 to 0.16 & 0.10 & * \\
\hline$\%$ Male & 0.22 & 0.06 to 0.37 & * & 0.15 & -0.002 to 0.30 & 0.02 & \\
\hline$\%$ Poverty & -0.25 & -0.28 to -0.21 & * & -0.08 & -0.15 to -0.01 & -0.04 & * \\
\hline Median household income (per $\$ 1000)$ & 0.01 & 0.01 to 0.02 & * & -0.00005 & -0.004 to 0.004 & 0.0005 & \\
\hline$\%$ No health insurance & -0.16 & -0.21 to -0.11 & * & -0.05 & -0.12 to 0.008 & -0.02 & \\
\hline$\% 65$ years and older & -0.14 & -0.20 to -0.07 & * & -0.03 & -0.10 to 0.04 & -0.009 & \\
\hline \multicolumn{8}{|l|}{ Population density } \\
\hline Urban & 0.00 & & & 0.00 & & & \\
\hline Not urban & 0.05 & 0.003 to 0.10 & * & -0.34 & -0.82 to 0.11 & -0.02 & \\
\hline
\end{tabular}

Coefficients are presented as a 10-unit change in the covariate unless otherwise specified.

*Statistical significance at the alpha 0.05 level.

†A 100-unit change.

$\ddagger$ A one-unit change.

CVHI, cardiovascular health index; FQHC, Federally Qualified Health Centre; HSA, health service area; RHC, Rural Health Centre.

Few studies examine how individual and community demographic factors may interact to modify associations between health and HCrRes. Our results suggest that individuals who derived the largest CVH benefit from increased PCP-S were middle age; females; non-Hispanic or 'other' race/ethnicity; those with a household income $<\$ 25000 /$ year; those living in a non-urban community; and those in communities with low insurance coverage. The increased benefit for individuals living in communities with low insurance coverage may be due to the presence of RHCs and FQHCs. In a brief subanalysis, we confirmed that RHCs and FQHCs are present in significantly higher numbers in these communities (data not shown). Further, this observation is consistent with studies suggesting that community health centres are particularly helpful to vulnerable populations. ${ }^{29}$ Groups benefiting the least from increased PCP-S were young adults and non-Hispanic blacks. In young adults, a negative association between CVHI and PCP-S may result from first-time healthcare visits resulting in a medical diagnosis (leading to decreased CVHI score). Our observation that non-Hispanic blacks do not benefit from increased PCP-S suggests that there may be other barriers to care or other factors affecting the relationship between HCrRes and health in this demographic group. Collectively, findings highlight the complex nature of the relationships between individuals and community-level and system-level variables. Future studies should better elucidate the complex relationships between PCP-S, medical homes, health seeking behaviour and health.

\section{Implications}

Our results suggest two possible mechanistic pathways that may underpin our observations. The first pathway, though not the focus of this study but which cannot be overlooked, is that of individual mechanisms. Specifically, our results suggest that improving individual-level CVH would require targeting modifiable 

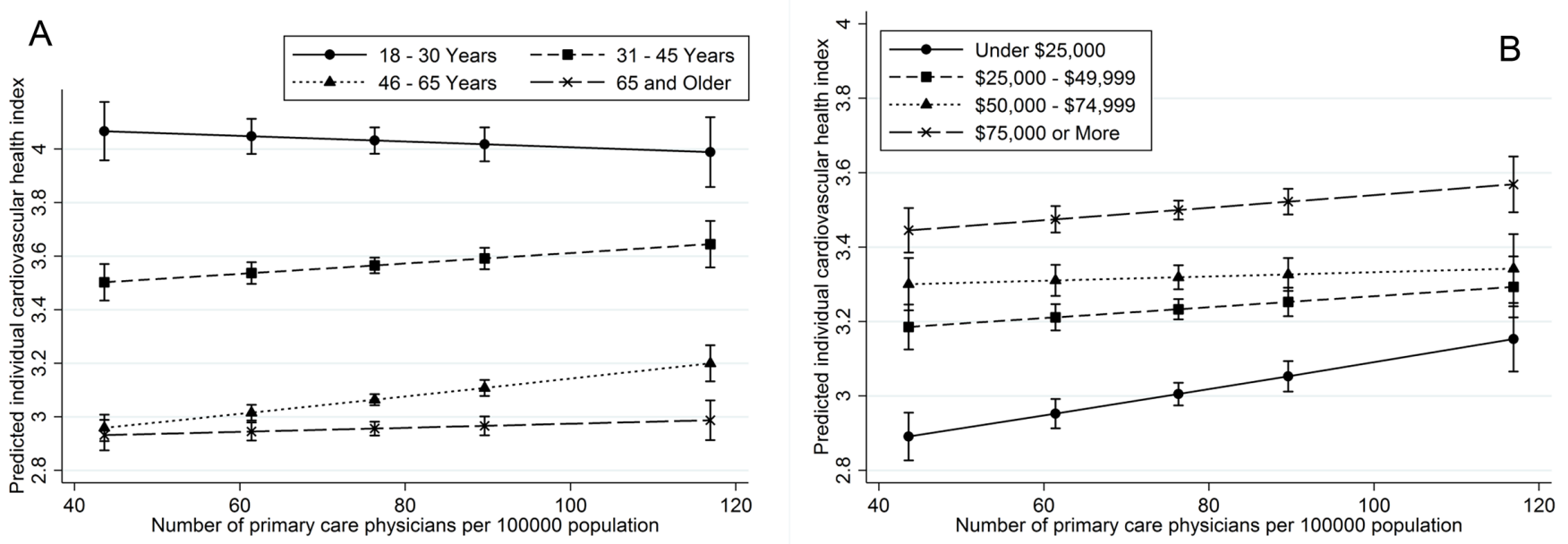

C
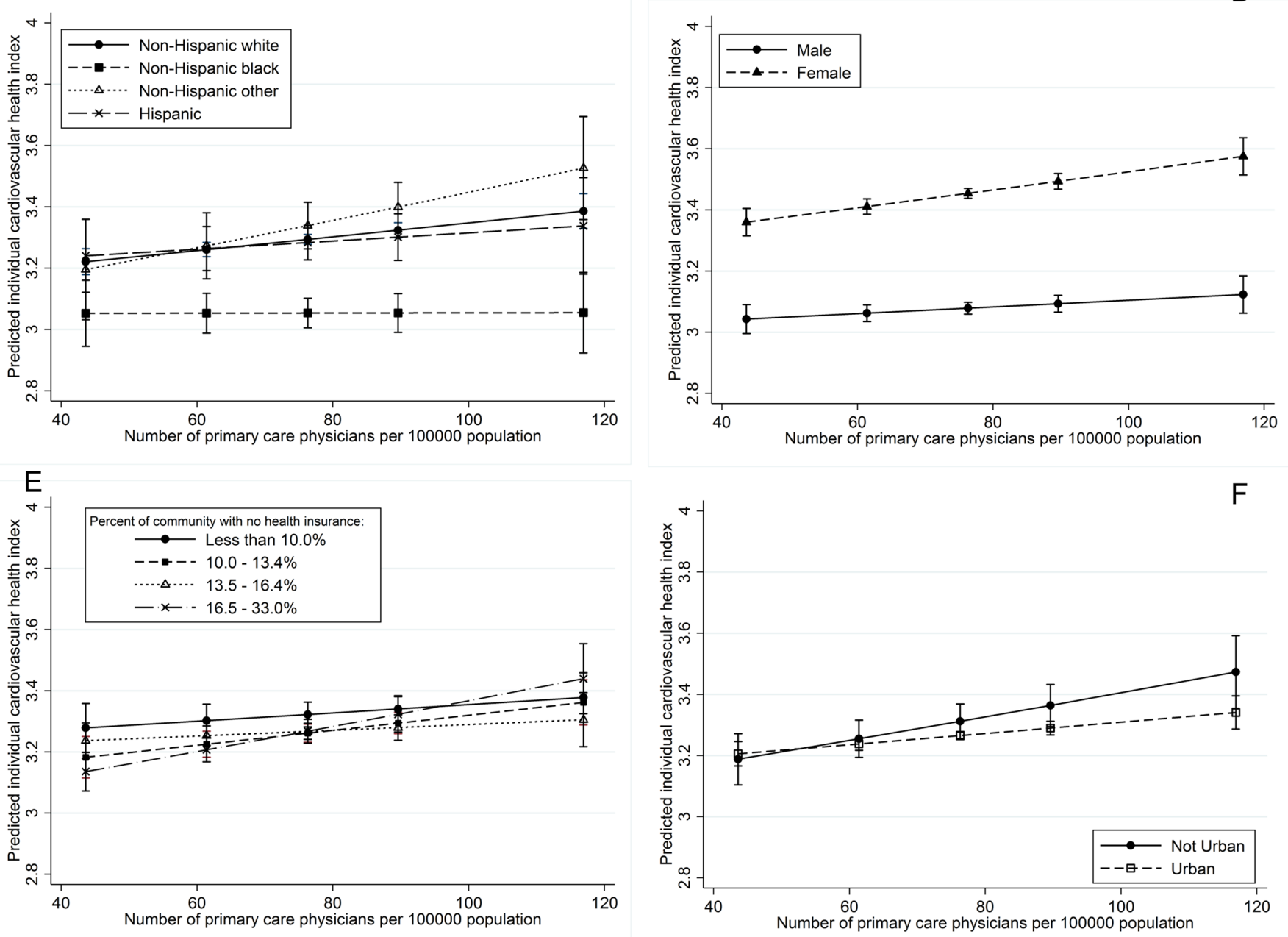

Figure 2 Results from regression analysis demonstrating differential association (interaction) between the number of primary care physicians per 100000 population and individual-level predicted cardiovascular health index based on individual and community (health service area) covariates (demographic and socioeconomic factors): (A) individual age; (B) individual income; (C) individual race/ethnicity; (D) individual sex; (E) community insurance coverage; and (F) community population density.

individual factors, particularly education and income. The continued importance of education for individual health cannot be ignored, and policies and programmes supporting higher educational attainment would likely result in improved health.
The second mechanistic pathway, and the primary focus of this study, is that of community-level healthcare resources, specifically the supply of primary care providers but not specialists or hospital beds. PCP-S was consistently associated with improved $\mathrm{CVH}$ in 
individuals, even after adjustment for all individual and community-level socioeconomic factors. PCP-S was also consistently associated with improved CVH in entire communities, ceteris paribus for all community-level socioeconomic factors. Given this observed, persistent importance of PCP-S, approaches to increase PCP-S would likely improve CVH in individuals and entire communities as a result of improved access to primary prevention and preventive healthcare services, including cardiovascular primary prevention and primary healthcare services targeted at CVD risk factor reduction. Thus, our results lend quantitative support to policies and programmes ${ }^{4810} 10$ aimed at increasing access to primary care in order to increase $\mathrm{CVH}$ and reduce disparities in $\mathrm{CVH}$ between age, income and gender groups.

However, the continued decline in the number of medical school graduates selecting primary care specialties presents a real and substantial challenge, especially in rural and underserved communities. ${ }^{42}$ The antiprimary care specialisation trend is also a concern for PAs and NPs, where only $43 \%$ of PAs and $52 \%$ of NPs choose primary care specialties. ${ }^{43}$ The magnitude of this challenge was underscored in a recent report that re-distribution of Graduate Medical Education (GME) payments from the Medicare Modernization Act, originally designed to increase medical residents in rural areas and in primary care, in fact had a net-negative effect on primary care training. ${ }^{44}$ Research is urgently needed to identify strategies that effectively and meaningfully impact the number of primary care providers, especially in underserved communities.

\section{Strengths and limitations}

A key strength of this study is the use of BRFSS-a large, nationally representative sample. The key, and well-recognised, limitation of this study is the self-report methodology for all BRFSS questions, which may have overestimated CVHI. However, previous studies have documented both differences and consistencies in national prevalence estimates between BRFSS and other national health surveys. ${ }^{45}$ Bias may have also resulted from our exclusion criteria: there was a significant proportion of participants with missing data and excluded communities were more likely to be rural with higher Hispanic populations. Thus, our results may not be generalisable to those populations.

\section{SUMMARY AND CONCLUSIONS}

This study provides an assessment of the specific healthcare resources that are associated with higher $\mathrm{CVH}$, and is unique in that we assessed the associations with $\mathrm{CVH}$ in both individuals and entire communities. An increased focus on strategies and policies that affect health in entire populations is of growing importance: continued focus on the identification of individual-level risk factors and interventions is neither realistic nor sustainable given the increasing prevalence of chronic disease and chronic disease risk factors. Our results provide strong support that increasing the supply of primary care providers is likely to improve CVH in individuals and entire communities. Our findings, however, note two key caveats: not all sociodemographic groups will be likely to benefit equally from additional primary care providers and there are significant challenges to increasing the number of primary care providers in communities.

Contributors All authors contributed to the conception and design of the study, and interpretation of the findings. CSP performed statistical analyses and drafted the manuscript. CSP, SSS, TKB and SJF contributed to the revision of the manuscript. All authors approved the final version.

Competing interests None declared.

Ethics approval This study was approved as non-human subjects research by the West Virginia University Institutional Review Board and as exempt by the University of Western Ontario Research Ethics Board.

Provenance and peer review Not commissioned; externally peer reviewed.

Data sharing statement № additional data are available.

Open Access This is an Open Access article distributed in accordance with the Creative Commons Attribution Non Commercial (CC BY-NC 4.0) license, which permits others to distribute, remix, adapt, build upon this work non-commercially, and license their derivative works on different terms, provided the original work is properly cited and the use is non-commercial. See: http://creativecommons.org/ licenses/by-nc/4.0/

(c) Article author(s) (or their employer(s) unless otherwise stated in the text of the article) 2017. All rights reserved. No commercial use is permitted unless otherwise expressly granted.

\section{REFERENCES}

1. Mozaffarian D, Benjamin EJ, As G, et al. Executive summary: heart disease and stroke statistics-2015 update: a report from the American Heart Association. Circulation 2015;131:434-41.

2. Heidenreich PA, Trogdon JG, Khavjou OA, et al. Forecasting the future of cardiovascular disease in the United States: a policy statement from the American heart association. Circulation 2011;123:933-44.

3. Lloyd-Jones DM, Hong Y, Labarthe D, et al. Defining and setting national goals for cardiovascular health promotion and disease reduction: the American Heart Association's strategic Impact Goal through 2020 and beyond. Circulation 2010;121:586-613.

4. U.S. Department of Health and Human Services. Healthy people 2020 objectives. 2013 http://www.healthypeople.gov/2020/ topicsobjectives2020/default

5. Association AH. My life check - live better with life's simple seven. http://www.heart.org/HEARTORG/Conditions/My-Life-Check---LifesSimple-7_UCM_471453_Article.jsp\#.WfcsGHZrxnl (accessed 12 Jul 2017).

6. Fang J, Yang Q, Hong Y, et al. Status of cardiovascular health among adult Americans in the 50 States and the District of Columbia, 2009. $J$ Am Heart Assoc 2012;1:e005371.

7. Shay CM, Ning H, Allen NB, et al. Status of cardiovascular health in US adults: prevalence estimates from the National Health and Nutrition Examination Surveys (NHANES) 2003-2008. Circulation 2012;125:45-56.

8. Mujahid MS, Moore LV, Petito LC, et al. Neighborhoods and racial/ ethnic differences in ideal cardiovascular health (the Multi-Ethnic Study of Atherosclerosis). Health Place 2017;44:61-9.

9. Huffman MD, Capewell S, Ning $\mathrm{H}$, et al. Cardiovascular health behavior and health factor changes (1988-2008) and projections to 2020: results from the National Health and Nutrition Examination Surveys. Circulation 2012;125:2595-602.

10. Pearson TA, Palaniappan LP, Artinian NT, et al. American Heart Association Guide for Improving Cardiovascular Health at the Community Level, 2013 update: a scientific statement for public health practitioners, healthcare providers, and health policy makers. Circulation 2013;127:1730-53.

11. Starfield B, Shi L, Macinko J. Contribution of primary care to health systems and health. Milbank Q 2005;83:457-502. 
12. Starfield B, Shi L, Grover A, et al. The effects of specialist supply on populations' health: assessing the evidence. Health Aff 2005;Suppl Web Exclusives:W5-97-W5-107.

13. Jasek JP. Having a primary care provider and receipt of recommended preventive care among men in New York City. Am J Mens Health 2011;5:225-35.

14. Blewett LA, Johnson PJ, Lee B, et al. When a usual source of care and usual provider matter: adult prevention and screening services. J Gen Intern Med 2008;23:1354-60.

15. Halm EA, Lee $C$, Chassin MR. Is volume related to outcome in health care? A systematic review and methodologic critique of the literature. Ann Intern Med 2002;137:511-20.

16. Cooper RA. States with more physicians have better-quality health care. Health Aff 2009;28:w91-w102.

17. Goodman DC, Grumbach K. Does having more physicians lead to better health system performance? JAMA 2008;299:335-7.

18. Gravelle H, Morris S, Sutton M. Are family physicians good for you? Endogenous doctor supply and individual health. Health Serv Res 2008;43:1128-44.

19. Bigbee JL. Relationships between nurse- and physician-topopulation ratios and state health rankings. Public Health Nurs 2008;25:244-52.

20. Shi L. The relationship between primary care and life chances. $J$ Health Care Poor Underserved 1992;3:321-35.

21. Macinko J, Starfield B, Shi L. Quantifying the health benefits of primary care physician supply in the United States. Int J Health Serv 2007;37:111-26.

22. Salsberg ES, Forte GJ. Trends in the physician workforce, 19802000. Health Aff 2002;21:165-73.

23. Moote M, Krsek C, Kleinpell R, et al. Physician assistant and nurse practitioner utilization in academic medical centers. Am J Med Qual 2011;26:452-60.

24. Bauer JC. Nurse practitioners as an underutilized resource for health reform: evidence-based demonstrations of cost-effectiveness. J Am Acad Nurse Pract 2010;22:228-31.

25. Dierick-van Daele AT, Metsemakers JF, Derckx EW, et al. Nurse practitioners substituting for general practitioners: randomized controlled trial. J Adv Nurs 2009;65:391-401.

26. Henry LR, Hooker RS, Yates KL. The role of physician assistants in rural health care: a systematic review of the literature. J Rural Health 2011;27:220-9.

27. Taylor J. Fundamentals of Community Health Centers. National Health Policy Forum. Paper 136. Washington DC: National Health Policy Forum, 2004 http://hsrc.himmelfarb.gwu.edu/sphhs_centers_ nhpf/136/

28. U.S. Department of Health and Human Services Health Resources Administration. What is a health center? 2014 http://bphc.hrsa.gov/ about/index.html

29. Shi L, Stevens GD. The role of community health centers in delivering primary care to the underserved: experiences of the uninsured and Medicaid insured. J Ambul Care Manage 2007;30:159-70.
30. Shi L, Lebrun LA, Hung LM, et al. US primary care delivery after the Health Center Growth Initiative: comparison of health centers, hospital outpatient departments, and physicians' offices. J Ambul Care Manage 2012;35:60-74

31. Laiteerapong N, Kirby J, Gao Y, et al. Health care utilization and receipt of preventive care for patients seen at federally funded health centers compared to other sites of primary care. Health Serv Res 2014;49:1498-518.

32. Centers for Disease Control and Prevention. Behavioral risk factor surveillance system annual survey data. 2014 https://www.cdc.gov/ brfss/index.html

33. Makuc DM, Haglund B, Ingram DD, et al. Health service areas for the United States. Vital Health Stat 1991:1-102.

34. National Cancer Institute SEER. Surveillance, epidemiology, and end result program: Health Service Areas (HSA). 2008 http://seer.cancer. gov/seerstat/variables/countyattribs/hsa.html

35. Pilkerton CS, Singh SS, Bias TK, et al. Changes in cardiovascular health in the United States, 2003-2011. J Am Heart Assoc 2015;4:e001650.

36. U.S. Department of Health and Human Services Health Resources and Services Sdministration. Area Health Resources File. 2013 https://datawarehouse.hrsa.gov/topics/ahrf.aspx

37. Klein RJ, Schoenborn CA. Age adjustment using the 2000 projected US population. Healthy People 2010 Stat Notes 2001 Jan;(20):1-10.

38. Everett CM, Schumacher JR, Wright A, et al. Physician assistants and nurse practitioners as a usual source of care. J Rural Health 2009;25:407-14.

39. Baker DP, Leon J, Smith Greenaway EG, et al. The education effect on population health: a reassessment. Popul Dev Rev 2011;37:307-32.

40. Sox CM, Swartz K, Burstin HR, et al. Insurance or a regular physician: which is the most powerful predictor of health care? Am J Public Health 1998;88:364-70.

41. McClurkin MA, Yingling LR, Ayers $C$, et al. Health insurance status as a barrier to ideal cardiovascular health for U.S. adults: data from the National Health and Nutrition Examination Survey (NHANES). PLoS One 2015;10:e0141534.

42. McCarthy M. US production of new primary care doctors falls far short of need, study finds. BMJ 2013;346:f4018.

43. Petterson SM, Phillips RL, Bazemore AW, et al. Relying on NPs and PAs does not avoid the need for policy solutions for primary care. Am Fam Physician 2013;88:230.

44. Chen C, Xierali I, Piwnica-Worms K, et al. The Redistribution of graduate medical education positions in 2005 failed to boost primary care Or rural training. Health Aff 2013;32:102-10.

45. Li C, Balluz LS, Ford ES, et al. A comparison of prevalence estimates for selected health indicators and chronic diseases or conditions from the Behavioral Risk Factor Surveillance System, the National Health Interview Survey, and the National Health and Nutrition Examination Survey, 2007-2008. Prev Med 2012;54:381-7. 